\title{
Research on demographic, clinical and paraclinical aspects in pregnant women infected with Toxoplasma gondii
}

\author{
ANDREI CSEP $^{1 *}$, LUMINIT,A LIGIA VAIDA ${ }^{2}$, BIANCA-MARIA NEGRUȚIU $^{2}$, BIANCA IOANA TODOR ${ }^{2 *}$, \\ CLAUDIA TEODORA JUDEA-PUSTA ${ }^{3 *}$, CAMELIA BUHAS $^{3}$ and CRISTIAN SAVA ${ }^{4}$ \\ Departments of ${ }^{1}$ Psycho-Neuroscience and Recovery, ${ }^{2}$ Dentistry, ${ }^{3}$ Morphological Disciplines, \\ ${ }^{4}$ Medical Disciplines, Faculty of Medicine and Pharmacy, University of Oradea, 410087 Oradea, Romania
}

Received September 10, 2021; Accepted October 11, 2021

DOI: $10.3892 /$ etm.2021.11046

\begin{abstract}
Toxoplasmosis is a zoonotic disease caused by the Toxoplasma gondii protozoan parasite. The aim of this study was to determine the frequency of acute toxoplasmosis or its presence in the medical history of 240 pregnant women, depending on age and geographical background. The second purpose was to analyze several medical aspects (clinical and paraclinical) in pregnant women with acute toxoplasmosis. The study identified several serological changes including positive toxoplasma $\operatorname{IgM}, \operatorname{IgG}$, and $\operatorname{Ig} \mathrm{A}$ antibodies. The chemiluminescence immunoassay (CLIA) method was used to detect T. gondii-specific $\operatorname{IgM}$ and $\operatorname{IgG}$ antibodies and the enzyme immunoassay (EIA) method to detect $T$. gondii-specific IgA antibodies. Of the 96 pregnant women (40\%) infected with T. gondii, approximately $1 / 3$ had acquired acute infection and $2 / 3$ had a history of prior acute infection $(\mathrm{P}<0.0001)$. No statistically significant differences according to age groups $(\mathrm{P}=0.9384)$ were found in terms of serological profile. The number of patients with negative serology was significantly higher in urban areas than in rural areas $(\mathrm{P}<0.0001)$. The highest incidence among the pregnant women with acute toxoplasmosis with a single chain of ganglia affected was represented by those with the involvement of cervical lymph nodes $(75 \%)$, the difference compared to the involvement of other chains of ganglia being statistically significant $(\mathrm{P}=0.0087)$. All 35 pregnant women with acute toxoplasmosis tested positive for $T$. gondii-specific IgM antibodies. Most pregnant women with acute $T$. gondii infection (57.1\%) tested
\end{abstract}

Correspondence to: Professor Luminița Ligia Vaida or Dr Bianca-Maria Negruțiu, Department of Dentistry, Faculty of Medicine and Pharmacy, University of Oradea, 1 Universitatii Street, 410087 Oradea, Romania

E-mail: ligia_vaida@yahoo.com

E-mail: biancastanis@yahoo.com

${ }^{*}$ Contributed equally

Key words: Toxoplasma gondii, pregnant women, T. gondii-specific IgM, IgG, IgA antibodies, lymphadenopathy negative for $T$. gondii-specific IgM antibody serum titres within 3-6 months of presentation. The difference compared to other intervals from the moment pregnant women sought care was statistically significant $(\mathrm{P}=00002)$. Only $80 \%$ of all pregnant women with acute toxoplasmosis tested positive for T. gondii-specific IgA antibodies. Pregnant women with acute toxoplasmosis have a 3.3 times higher risk of pregnancy loss.

\section{Introduction}

Toxoplasmosis is a zoonotic disease caused by the Toxoplasma gondii (T. gondii) protozoan parasite $(1,2)$. Toxoplasmosis is widespread and it is present in every country and seropositivity rates range from less than $10 \%$ to over $90 \%$ (3). It is estimated that onethird of the world's human population is infected by this parasite $(4,5)$. Human infection results often from the ingestion or handling of undercooked meat of intermediate hosts such as sheep, pigs and birds containing tissue cysts. In other cases, it may result from direct contact with cats or from the consumption of water or food contaminated by oocysts excreted in the feces of infected cats $(2,6)$. Toxoplasmosis is typically an asymptomatic infection resulting in life-long latent infection in healthy individuals (7). However, this infection is an opportunistic one that can cause severe complications in immune-suppressed diseased individuals such as AIDS patients (8-11). Several studies that have investigated the human population infected by $T$. gondii suggest that this pathogen may play an etiological role in some mental and behavioral disorders (12-14). Women infected with $T$. gondii before pregnancy usually do not transmit the parasite to their fetuses. Acute toxoplasma infection during pregnancy can lead to congenital toxoplasmosis for the fetus and newborn. It can cause abortion, intrauterine fetal demise and syndromes that include neurologic and neurocognitive deficits and chorioretinitis $(15,16)$.

Several studies have demonstrated that some environmental factors, whether occurring as a natural phenomenon or through human intervention, can strongly influence the occurrence, transmission and distribution of $T$. gondii, especially climate, today's rapid urbanization, global warming, and economic globalization $(7,17,18)$. Other important predictors of $T$. gondii infection are considered: sociodemographic factors, behavioral and obstetric factors, geographic location, presence 
of cats in the household, agriculture practice (exposure to contaminated soil through farming or gardening barehanded), history of spontaneous abortion and maternal age $(2,7,19)$.

Oocysts are resistant to the environment. They can sporulate in water and become infectious to their hosts, surviving for a period of up to 6 months in seawater $(20,21)$. It has been suggested that human infection caused by oocysts is usually more severe than that caused by the ingestion of tissue cysts, regardless of the dose (22).

\section{Patients and methods}

Participants. The study included 240 pregnant women who were referred to the Infectious Diseases Office from 'Dr. Gavril Curteanu' Municipal Clinical Hospital, Oradea and to the Infectious Diseases Outpatient Clinic from the County Emergency Clinical Hospital, Oradea, Romania during 01/01/2016 to 31/12/2019. The study was conducted in accordance with the World Medical Association (WMA) Declaration of Helsinki, Ethical Principles for Medical Research Involving Human Subjects, and approved by the Ethics Committee of the University of Oradea, Romania (project identification code: 17/22.01.2021).

Particular attention was paid to the inclusion, respectively exclusion criteria in the study. The inclusion criteria were: i) pregnant women aged between 18 and 40 years; ii) request to perform serological diagnosis in order to determine the immune status for this parasite; iii) presence of at least one of the following criteria at presentation: pregnant women with clinical signs specific to $T$. gondii infection with lymphadenopathy, pregnant women with a history of pregnancy loss prior to the current pregnancy, pregnant women who kept pets (cats) at home; iv) pregnant women who could be kept under medical observation for 1 year in order to monitor in dynamics the Toxoplasma IgM, IgA, IgG antibody titters; v) stable residence in Bihor County.

The exclusion criteria were: i) pregnant women under 18 years of age and over 40 years of age; ii) pregnant women who refused clinical and serological monitoring for 1 year to capture seroconversion.

Methods. The first stage of the study consisted in describing the group of pregnant women in terms of age groups as well as in terms of geographical background (urban/rural). The next stage of the study aimed to identify several serological changes: pregnant women with acute toxoplasmosis tested positive for toxoplasma IgM and IgG antibodies, positive/negative for toxoplasma $\operatorname{IgA}$ antibodies, their evolution being followed in dynamics; pregnant women with a history of acute infection tested negative for toxoplasma IgM antibodies and positive for toxoplasma IgG antibodies. In the case of pregnant women diagnosed with acute toxoplasmosis, IgG avidity test was also performed in order to established precisely the moment of infection.

Serological assay. Blood samples to determine the serum level of $T$. gondii-specific $\operatorname{IgM}, \operatorname{IgA}$ and $\operatorname{IgG}$ antibodies were drawn in several stages: the first test at the patient's presentation (onset of the disease); the following test at 4 weeks; then at 3, 6, 9 and 12 months from the initial sample in order to monitor in dynamics the T. gondii-specific $\operatorname{IgM}, \operatorname{IgA}$ and $\operatorname{IgG}$ antibody titres.

Determination of IgM and IgG toxoplasma antibodies were performed in Biostandard Laboratories by the chemiluminescence immunoassay (CLIA) method. For this we used the Immulite 2000 analyzer (Siemens), together with the necessary reagents, produced also by Seimens.

Detection of $T$. gondii-specific IgA antibodies was carried out by the Bioclinica Laboratories, using the Chorus analyzer (Diesse Diagnostica Senese S.p.A). The used method was enzyme immunoassay (EIA).

The IgG avidity test was also carried out by the Bioclinica Laboratories, using the Biomerieux Vidas analyzer and reagents. The method used was enzyme linked fluorescent assay (ELFA). An index $<0.200$ indicates low avidity. An index between 0.200 and 0.300 indicates intermediate avidity, and an index $>0.300$ indicates high avidity, showing that the primary infection occurred 4 months before.

Following, the study presented the correlation between the serological profile of the respective pregnant women and their age, respectively their geographical background.

Next, the pregnant women diagnosed with acute toxoplasmosis were examined in terms of correlative demographic parameters, respectively in terms of the medical aspects, the frequency of lymphadenopathy, the risk of developing lymphadenopathy, the location of the lymphadenopathy and its degree of extension, as well as possible correlations with patient age were assessed.

In the next stage, we performed serological examinations, determining in all pregnant women the toxoplasma $\operatorname{IgM}, \operatorname{IgG}$, and IgA antibody titres as well as their dynamic evolution over a period of 12 months, namely: in the first month, at 1-3 months, at 3-6 months, at 6-9 months, at 9-12 months. The IgG avidity test was carried out in patients with acute toxoplasmosis to determine as accurately as possible the timing of infection.

Another stage of the research conducted a comparative monitoring of the course of pregnancy in pregnant women with acute toxoplasmosis vs. pregnant women without acute toxoplasmosis in terms of onset, risk of miscarriage in pregnant women with acute toxoplasmosis and frequency of stillbirths.

Data analysis. MedCalc ${ }^{\circledR}$ version 9.4.2.0 (MedCalc ${ }^{\circledR}$ Software) statistical software was used to store the information from the patients' medical records in a database and to perform statistical calculations. The results of the statistical tests shall be represented by the probability of the 'null' hypothesis (P), its value below 0.05 proves a statistically significant difference between the studied groups. Certain results shall be presented graphically using the same statistical software. Microsoft ${ }^{\circledR}$ Excel $^{\circledR} 2010$ (Microsoft ${ }^{\circledR}$ Corporation, USA) was used for several charts. The Kolmogorov-Smirnov test was used to assess each continuous variable for value distribution compared to the normal population. Non-parametric tests were conducted (for variables with asymmetric distribution) depending on the variable. The non-parametric methods used during the study were: the Mann-Whitney test where there are only two study groups. Categorical variables are described by their absolute values and percentages in parentheses. They were studied using the following tests: the Chi-square test with Yates' correction for continuity-in the case of $2 \times 2$ contingency 
Table I. Distribution by the presence of lymphadenopathy of pregnant women with acute forms of disease and of pregnant women without acute forms of disease.

Pregnant women

\begin{tabular}{|c|c|c|c|}
\hline \multirow[b]{2}{*}{ Lymphadenopathy } & & \multirow[b]{2}{*}{$\mathrm{n}(\%)$} \\
\hline & $\begin{array}{c}\text { Without acute toxoplasmosis } \\
\mathrm{n}\end{array}$ & $\begin{array}{l}\text { With acute toxoplasmosis } \\
n\end{array}$ & \\
\hline No lymphadenopathy & 60 & 4 & $64(26.7 \%)$ \\
\hline With lymphadenopathy & 145 & 31 & $\begin{array}{c}176(73.3 \%) \\
(\mathrm{P}=0.0456)\end{array}$ \\
\hline Total \% & $205(85.4 \%)$ & $35(14.6 \%)$ & $240(100 \%)$ \\
\hline
\end{tabular}

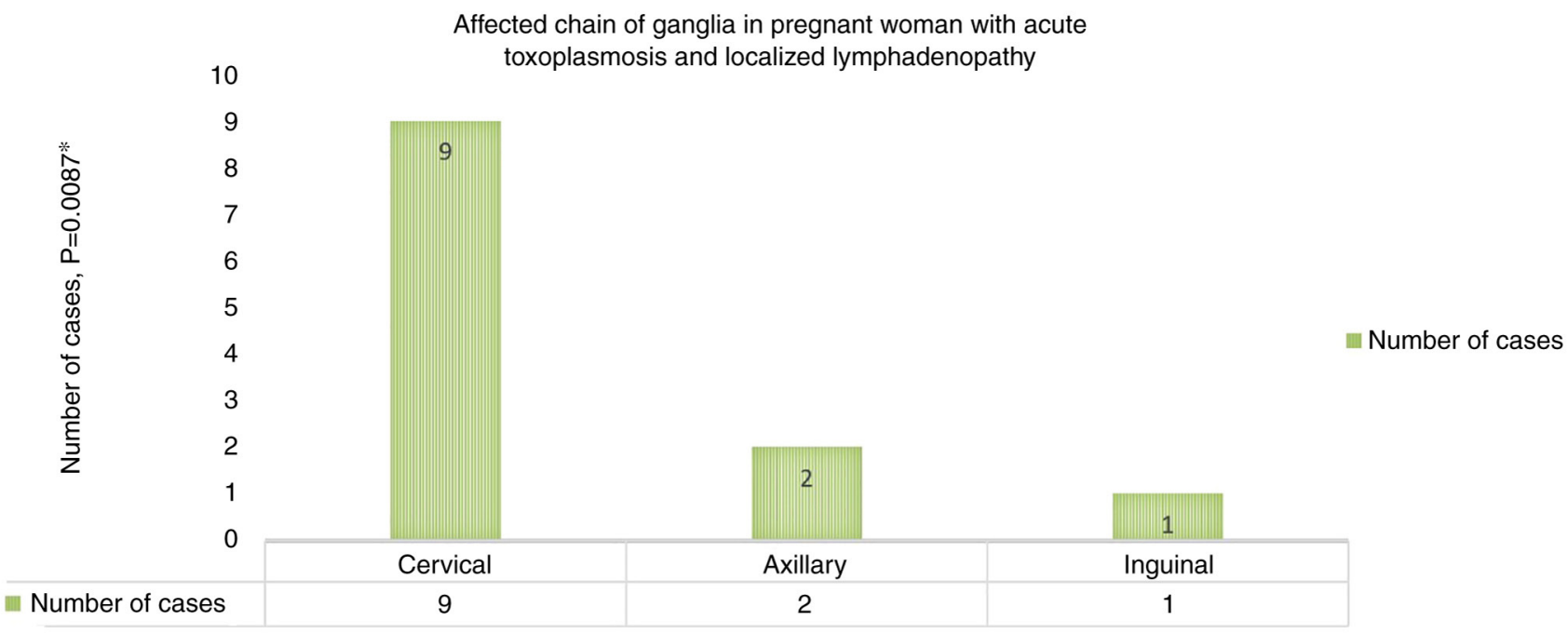

Figure 1. The affected chain of ganglia in pregnant women with acute toxoplasmosis with localized lymphadenopathy.

tables (categorical variables with 2 possible values between 2 study groups) with a number of cases over 20 ; simple Chi-square test-for other types of frequency tables $(3 \times 2,3 \times 3)$.

\section{Results}

The pregnant women included in the study showed an increased share of the age groups $21-25$ years [86 cases $(35.8 \%)$ ] and 26-30 years [62 cases $(25.8 \%)$ ], the difference compared to the other age groups being statistically significant $(\mathrm{P}<0.0001)$.

The pregnant women included in the study came mainly from rural areas [137 cases (57.1\%)] compared to urban areas [103 cases $(42.9 \%)]$, the difference being statistically significant $(\mathrm{P}=0.0332)$.

Of the 240 pregnant women monitored during the four years of study, $60 \%$ (144 cases) did not show serological changes specific to T. gondii infection. Of the 96 pregnant women (40\%) infected with $T$. gondii, approximately $1 / 3$ [35 cases (14.6\%)] had acquired acute infection and 2/3 [61 cases (25.4\%)] had a history of prior acute infection $(\mathrm{P}<0.0001)$.

The share of pregnant women with acute toxoplasmosis in the four years studied was $14.6 \%$ (35 cases). The distribution of the reference pregnant women by study years did not show statistically significant differences $(P=0.8563)$. In addition, there were no statistically significant differences in the distribution of pregnant women by age groups $(\mathrm{P}=0.0997)$, by years and by age groups $(\mathrm{P}=0.4410)$, by region of origin $(\mathrm{P}=0.3105)$ even if the pregnant women from rural areas were in a higher number, or by the region of origin and years of study $(\mathrm{P}=0.4804)$.

Pregnant women with acute toxoplasmosis showed lymphadenopathy more frequently [31 cases $(88.57 \%)$ ] than pregnant women without acute toxoplasmosis in the study group [145 cases $(70.73 \%)$ ] (Table I), the difference being statistically significant $(\mathrm{P}=0.0456)$. The relative risk of developing lymphadenopathy in pregnant women with acute toxoplasmosis was 1.2522 higher than in pregnant women without acute toxoplasmosis.

There was no statistically significant difference between localized or generalized lymphadenopathy caused by the presence or absence of acute toxoplasmosis among the reference pregnant women $(\mathrm{P}=0.8462)$. In addition, the presence or absence of lymphadenopathy was not correlated with the age groups $(\mathrm{P}=0.0581)$. The localized or generalized character of the lymphadenopathy was not correlated with the age groups either $(\mathrm{P}=0.8537)$.

The highest incidence among the pregnant women with acute toxoplasmosis with a single chain of ganglia affected (Fig. 1) was represented by those with the involvement of cervical lymph nodes [ 9 cases $(75 \%)$ ], the difference compared 
Table II. Distribution of the course of pregnancy in women with present or absent acute toxoplasmosis.

\begin{tabular}{lccc}
\hline & \multicolumn{3}{c}{ Acute toxoplasmosis } \\
\cline { 2 - 3 } Course of pregnancy & Absent & Present & $\mathrm{n}$ \\
\hline Termination on request & $\mathrm{n}$ & $1 \%)$ & $1(0.4 \%)$ \\
Birth & 0 & 24 & $200(83.3 \%)$ \\
Birth of a stillborn fetus & 176 & 1 & $1(0.4 \%)$ \\
Premature birth & 0 & 0 & $4(1.7 \%)$ \\
Spontaneous pregnancy loss & 4 & 9 & $34(14.2 \%)$ \\
Total \% & 25 & $35(14.6 \%)(\mathrm{P}=0.0072)$ & $240(100 \%)$ \\
\hline
\end{tabular}

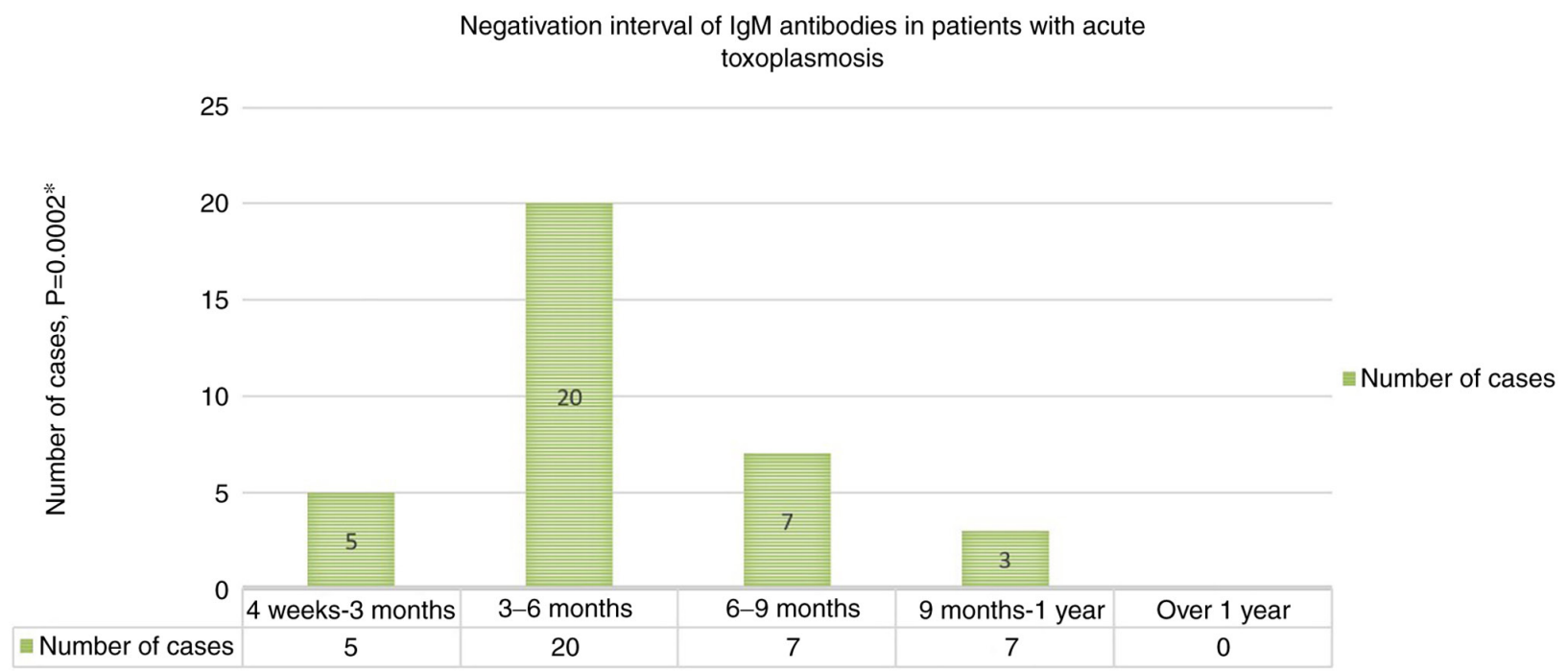

Figure 2. Negativation interval of $\operatorname{IgM}$ antibodies in patients with acute toxoplasmosis.

to the involvement of other chains of ganglia being statistically significant $(\mathrm{P}=0.0087)$.

The involvement of the cervical-axillary-inguinal lymph nodes at the same time [12 cases $(63.1 \%)$ ] was more common compared to the other combinations (cervical-axillary, cervical-inguinal, axillary-inguinal) $(\mathrm{P}=0.0013)$ in pregnant women with acute toxoplasmosis and generalized lymphadenopathy.

All 35 pregnant women with acute toxoplasmosis tested positive for T. gondii-specific IgM antibodies.

Most pregnant women with acute $T$. gondii infection [20 cases $(57.1 \%)]$ tested negative for $T$. gondii-specific IgM antibody serum titres within 3-6 months of presentation (Fig. 2). The difference compared to the other shorter (4 weeks-3 months) or longer intervals (6-9 months, 9-12 months) from the moment pregnant women sought care was statistically significant $(\mathrm{P}=0.0002)$.

No pregnant women in the studied group tested positive for $\operatorname{IgM}$ antibodies for more than 1 year from the time of diagnosis.

Only 28 cases $(80 \%)$ of all pregnant women with acute toxoplasmosis tested positive for $T$. gondii-specific IgA antibodies. In the remaining 7 cases $(20 \%)$, the $T$. gondii-specific
IgA antibodies had equivocal value. These antibodies were most frequently negative within 4 weeks to 3 months [19 cases $(75 \%)$ from the moment pregnant women sought care $(\mathrm{P}=0.0001)$.

Monitoring the course of pregnancy in women with acute toxoplasmosis compared to women without acute toxoplasmosis (Table II) indicated that the course of pregnancy may be directly correlated with the presence of acute $T$. gondii infection $(\mathrm{P}=0.0072)$. According to this study, pregnant women with acute toxoplasmosis had a 3.3 times higher risk of pregnancy loss than pregnant women without acute toxoplasmosis.

In $85 \%$ of the pregnant women with acute toxoplasmosis who tested positive for $T$. gondii-specific IgM, IgG, and IgA antibodies and who showed increased $\mathrm{IgG}$ avidity, the course of pregnancy was normal. Five percent of the pregnant women belonging to the respective serological profile underwent therapeutic abortion, suffered spontaneous abortion, or gave birth to a stillborn fetus, in the same proportions. All pregnant women who tested positive for $T$. gondii-specific $\operatorname{IgM}, \operatorname{IgG}$ and $\operatorname{Ig}$ A antibodies and showed low IgG avidity suffered a spontaneous pregnancy loss. The course of pregnancy was normal in pregnant women who tested positive for T. gondii-specific IgM and $\operatorname{IgG}$ antibodies, negative for 
T. gondii-specific IgA antibodies and showed increased IgG avidity.

\section{Discussion}

In the present study, we considered that pregnant women, especially those with an obstetric history, marked by pregnancy loss, spontaneous abortion, stillbirth, preterm birth, but also pregnant women who develop a clinical picture more or less suggestive for $T$. gondii infection should undergo a thorough examination. We started from the premise that investigations performed in the first trimester of pregnancy are useful to avoid the embryopathic effect of a possible infection. Based on Suzuki et al, we considered the fact that several elaborate examinations on specific immunology may determine the infection age and may also establish a certain therapeutic conduct. In their study related to several serological techniques used for diagnosis of acute acquired toxoplasmosis, the authors found that determination of the avidity of $T$. gondii-specific IgG by the titration method in patients with detectable $\operatorname{IgM}$ antibodies defines most accurately the stage of infection by $T$. gondii (23).

We refer to a selected group in the sense that each of the pregnant women included in our study presented at least one risk factor for $T$. gondii infection, these factors being decisive for requesting the investigation of the reference pregnant women in the special ward of the Infectious Diseases Clinic from Oradea and in the Infectious Diseases Outpatient Clinic from the County Emergency Clinical Hospital, Oradea, Romania.

The existence of these very restrictive inclusion criteria makes it difficult to compare our results with the values established by other studies which either address all pregnant women in a certain geographical area or in certain groups of pregnant women selected based on a specific obstetric pathology.

Our study demonstrated that serum titre for T. gondii-specific IgM antibodies was most frequently negative within 3-6 months from the moment of seeking care $(57.1 \%)$. The difference compared to the other shorter or longer intervals from the moment pregnant women sought care was statistically significant $(\mathrm{P}=0.0002)$. Only $80 \%$ of the pregnant women with acute toxoplasmosis tested positive for T. gondii-specific IgA antibodies. Patients tested negative for these antibodies most frequently within 4 weeks to 3 months from the time pregnant women sought care, faster than for T. gondii-specific IgM antibodies; the difference compared to the other intervals being statistically significant $(\mathrm{P}=0.0001)$. Monitoring the course of pregnancy in women with acute toxoplasmosis compared to women without acute toxoplasmosis shows that pregnancy loss may be directly correlated with the presence of acute $T$. gondii infection $(\mathrm{P}=0.0072)$. Pregnant women with acute toxoplasmosis had a 3.3 times higher risk of pregnancy loss than those without acute toxoplasmosis.

According to several data from the literature consulted as well, the increased prevalence of $T$. gondii infection in pregnant women from rural areas may be explained, to a large extent, by the increased presence of household pets (cats) which are not under the supervision of a veterinarian, with the possibility of easier spread of $T$. gondii oocysts in the external environment, becoming infective to pregnant women, as well as by the more frequent consumption of possibly insufficiently cooked food or insufficiently washed vegetables and fruit on which oocysts may exist. Accurate information on the quality of water and soil should increase people's awareness of the importance and the role of water for human health $(24,25)$.

Our study showed that $80 \%$ of all pregnant women with acute toxoplasmosis tested positive for $T$. gondii-specific IgA antibodies. This value is lower than the values reported by other authors, where $95 \%$ of the acquired toxoplasmosis recorded positive values of $T$. gondii-specific IgA antibodies (26). What is specific to our case study is that all women with acute toxoplasmosis tested negative for $T$. gondii-specific IgA antibodies within 9 months after presentation.

Medical advice regarding termination of the pregnancy in women suspected of being infected with $T$. gondii should consider not only the positivity for $T$. gondii-specific IgM, IgG, and $\operatorname{IgA}$ antibodies, but also the results of the IgG avidity test performed in the first trimester of pregnancy whose increased value indicates an infection prior to conception. Under these circumstances, there is certainty that the conception product shall not be infected, thus there shall be no cases of congenital toxoplasmosis $(27,28)$. In recent studies on the global prevalence of ATI in pregnant women infected with $T$. gondii, it was reported that the global prevalence of ATI was $1.1 \%$ while the studies that used strict criteria (seroconversion and low IgG avidity) for the definition of ATI showed that $0.6 \%$ of pregnant women had ATI during gestation. Thus, the prevalence of ATI varied due to many factors such as income level, human development indices and geographical location $(29,30)$.

Negative serum serology (both $\operatorname{IgG}$ and $\operatorname{IgM}$ ) is adequate to rule out $T$. gondii infection in immunocompetent patients. Even if serum serology has been widely used for the diagnosis of toxoplasmosis in both immunocompetent and immunocompromised patients, some seronegative clinical cases have been reported especially in immunocompromised patients. Complimentary investigations can be helpful in these atypical cases (31-34).

A study carried out by several Chinese authors showed that anti-T. gondii-specific IgG antibodies were found in $15.2 \%$ of pregnant women; $2.9 \%$ of pregnant women were positive for anti-T. gondii IgM antibodies. Among pregnant women, $12.6 \%$ were positive for $\operatorname{IgG}$ antibodies and $0.3 \%$ of pregnant women were positive for IgM antibodies only, while $2.6 \%$ pregnant women were positive for both $\operatorname{IgG}$ and $\operatorname{IgM}$ antibodies. Univariate analysis of sociodemographic and risk factors for pregnant women identified some factors with a value $\leq 0.25$ that may be related to infection. Four of these were found to be significantly associated with $T$. gondii infection: area of residence, cats in home, contact with cats and dogs, and exposure to soil (35).

There is little information about the epidemiology of T. gondii infection in pregnant women in Romania. According to some results, $T$. gondii seroprevalence in pregnant women has decreased from 43.79 to $38.81 \%$ in the last 10 years in the Western Region of Romania. Our study, unlike other recent studies carried out in Romania, did not show statistically significant differences related to the age of the pregnant women infected with $T$. gondii,while the prevalence of infections was higher in urban areas $(36,37)$. Consistent with the two studies mentioned above, several authors claim that there is a decreasing trend in $T$. gondii infection among pregnant 
women (38). Epidemiological studies conducted on $T$. gondii seroprevalence in pregnant women before the 2000's revealed rates ranging from $40.4 \%$ to $48.7 \%$ in two areas of Italy, whereas some recent studies have found rates of $T$. gondii prevalence in pregnant women between 21.5 and $27.5 \%$ (39-42).

In conclusion, serum titre for $T$. gondii-specific $\operatorname{IgM}$ antibodies was most frequently negative within 3-6 months from the moment of seeking care; $80 \%$ of the pregnant women with acute toxoplasmosis tested positive for $T$. gondii-specific IgA antibodies. Pregnant women with acute toxoplasmosis had a 3.3 times higher risk of pregnancy loss than those without acute toxoplasmosis.

\section{Acknowledgements}

We would like to thank our collaborators from Bioclinica and Biostandard Laboratories.

\section{Funding}

No funding was received.

\section{Authors' contributions}

$\mathrm{AC}, \mathrm{BMN}, \mathrm{CTJP}$ and $\mathrm{CB}$ were involved in the conception of the study and the interpretation of the data. AC, LLV, BIT and CS contributed to the acquisition of the data and performed statistical analysis. AC performed serological testing. AC, LLV and BMN wrote the manuscript. CTJP, CB, BIT and CS revised the manuscript for important intellectual content. All authors read and approved the final manuscript for publication.

\section{Availability of data and materials}

The datasets used and/or analyzed during the present study are available from the first author on reasonable request.

\section{Ethics approval and consent to participate}

This study was approved by the Ethics Committee of the University of Oradea, Faculty of Medicine and Pharmacy, Romania (project identification code: 17/22.01.2021). Informed consent was obtained from each patient.

\section{Patient consent for publication}

Not applicable.

\section{Competing interests}

The authors declare that they have no competing interests.

\section{References}

1. Tenter AM,Heckeroth AR and Weiss LM: Toxoplasma gondii: From animals to humans. Int J Parasitol 30: 1217-1258, 2000 .

2. Al-Adhroey AH, Mehrass AAO, Al-Shammakh AA, Ali AD Akabat MYM and Al-Mekhlafi HM: Prevalence and predictors of Toxoplasma gondii infection in pregnant women from Dhamar, Yemen. BMC Infect Dis 19: 1089, 2019.
3. Pappas G, Roussos $\mathrm{N}$ and Falagas ME: Toxoplasmosis snapshots: Global status of Toxoplasma gondii seroprevalence and implications for pregnancy and congenital toxoplasmosis. Int J Parasitol 39: 1385-1394, 2009.

4. Montoya JG and Liesenfeld O: Toxoplasmosis. Lancet 363: 1965-1976, 2004.

5. Torgerson PR and Mastroiacovo P: The global burden of congenital toxoplasmosis: A systematic review. Bull World Health Organ 91: 501-508, 2013.

6. Montoya JG and Remington JS: Management of Toxoplasma gondii infection during pregnancy. Clin Infect Dis 47: 554-566, 2008.

7. Yan C, Liang LJ, Zheng KY and Zhu XQ: Impact of environmental factors on the emergence, transmission and distribution of Toxoplasma gondii. Parasit Vectors 9: 137, 2016.

8. Tegegne D, Abdurahaman M, Mosissa T and Yohannes M: Anti-toxoplasma antibodies prevalence and associated risk factors among HIV patients. Asian Pac J Trop Med 9: 460-464, 2016.

9. Munoz M, Liesenfeld $\mathrm{O}$ and Heimesaat MM: Immunology of Toxoplasma gondii. Immunol Rev 240: 269-285, 2011.

10. Csep A, Vaida L, Bungau S and Todor BI: Clinical and biological correlations in Toxoplasma gondii infection in HIV immune suppressed diseased persons. Iran J Public Health 44: 1012-1013, 2015.

11. Pereira-Chioccola VL, Vidal JE and Su C: Toxoplasma gondii infection and cerebral toxoplasmosis in $\mathrm{HIV}$-infected patients. Future Microbiol 4: 1363-1379, 2009.

12. Carruthers VB and Suzuki Y: Effects of Toxoplasma gondii infection on the brain. schizophr. Bull 33: 745-751, 2007.

13. Webster JP, Lamberton PH, Donnelly CA and Torrey EF: Parasites as causative agents of human affective disorders? The impact of anti-psychotic, mood-stabilizer and anti-parasite medication on Toxoplasma gondii's ability to alter host behaviour. Proc Biol Sci 273: 1023-1030, 2006.

14. Fekadu A, Shibre T and Cleare AJ: Toxoplasmosis as a cause for behaviour disorders-overview of evidence and mechanisms. Folia Parasitol (Praha) 57: 105-113, 2010.

15. Jones JL, Lopez A, Wilson M, Schulkin J and Gibbs R: Congenital toxoplasmosis: A review. Obstet Gynecol Surv 56: 296-305, 2001

16. Kravetz JD and Federman DG: Toxoplasmosis in pregnancy. Am J Med 118: 212-216, 2005.

17. Mazzillo FF, Shapiro K and Silver MW: A new pathogen transmission mechanism in the ocean: The case of sea otter exposure to the land-parasite Toxoplasma gondii. PLoS One 8: e82477, 2013.

18. Afonso E, Germain E, Poulle ML, Ruette S, Devillard S, Say L, Villena I, Aubert D and Gilot-Fromont E: Environmental determinants of spatial and temporal variations in the transmission of Toxoplasma gondii in its definitive hosts. Int J Parasitol Parasites Wildl 2: 278-285, 2013.

19. Elmore SA, Jones JL, Conrad PA, Patton S, Lindsay DS and Dubey JP: Toxoplasma gondii: Epidemiology, feline clinical aspects, and prevention. Trends Parasitol 26: 190-196, 2010.

20. Lindsay DS, Collins MV, Mitchell SM, Cole RA, Flick GJ, Wetch CN, Lindquist A and Dubey JP: Sporulation and survival of Toxoplasma gondii oocysts in seawater. J Eukaryot Microbiol 50 (Suppl): S687-S688, 2003.

21. Ribeiro LA, Santos LK, Brito PA Jr, Maciel BM, Da Silva AV and Albuquerque GR: Detection of Toxoplasma gondii DNA in Brazilian oysters (Crassostrea rhizophorae). Genet Mol Res 14: 4658-4665, 2015

22. Jones JL and Dubey JP: Waterborne toxoplasmosis-recent developments. Exp Parasitol 124: 10-25, 2010.

23. Suzuki LA, Rocha RJ and Rossi CL: Evaluation of serological markers for the immunodiagnosis of acute acquired toxoplasmosis. J Med Microbiol 50: 62-70, 2001.

24. Babaie J, Amiri S, Mostafavi E, Hassan N, Lotfi P, Esmaeili Rastaghi AR and Golkar M: Seroprevalence and risk factors for Toxoplasma gondii infection among pregnant women in Northeast Iran. Clin Vaccine Immunol 20: 1771-1773, 2013.

25. Sărmăşan C, Drăghici S and Daina L: Identification, communication and management of risks relating to drinking water pollution in bihor county. EEMJ 7: 769-774, 2008.

26. Bessierès MH, Roques C, Berrebi A, Barre V, Cazaux M and Séguéla JP: IgA antibody response during acquired and congenital toxoplasmosis. J Clin Pathol 45: 605-608, 1992.

27. Lappalainen $M$ and Hedman K: Serodiagnosis of toxoplasmosis. The impact of measurement of IgG avidity. Ann Ist Super Sanita 40: 81-88, 2004. 
28. Horváth KN, Szénási Z, Danka J and Kucsera I: Value of the IgG avidity in the diagnosis of recent toxoplasmosis: A comparative study of four commercially available anti-Toxoplasma gondii IgG avidity assays. Acta Parasitol 50: 255-260, 2005.

29. Rostami A, Riahi SM, Contopoulos-Ioannidis DG, Gamble HR, Fakhri Y, Shiadeh MN, Behniafar H, Taghipour A, Maldonado YA, Mokdad AH, et al: Acute toxoplasma infection in pregnant women worldwide: A systematic review and meta-analysis. PLoS Negl Trop Dis 13: e0007807, 2019.

30. Nogareda F, Le Strat Y, Villena I, De Valk H and Goulet V: Incidence and prevalence of Toxoplasma gondii infection in women in France, 1980-2020: Model-based estimation. Epidemiol Infect 142: 1661-1670, 2014.

31. Yates WB, Chiong F, Zagora S, Post JJ, Wakefield D and McCluskey P: Ocular toxoplasmosis in a tertiary referral centre in Sydney Australia-clinical features, treatment, and prognosis. Asia Pac J Ophthalmol (Phila) 8: 280-284, 2019.

32. Ozgonul C and Besirli CG: Recent developments in the diagnosis and treatment of ocular toxoplasmosis. Ophthalmic Res 57: 1-12, 2017.

33. Garweg JG, De Groot-Mijnes JD and Montoya JG: Diagnostic approach to ocular toxoplasmosis. Ocul Immunol Inflamm 19: 255-261, 2011

34. Sigle M, El Atrouni W and Ajlan RS: Seronegative ocular toxoplasma panuveitis in an immunocompetent patient. Am J Ophthalmol Case Rep 19: 100745, 2000.

35. Cong W, Dong XY, Meng QF, Zhou N, Wang XY, Huang SY, Zhu XQ and Qian AD: Toxoplasma gondii infection in pregnant women: A seroprevalence and case-control study in Eastern China. Biomed Res Int 2015: 170278, 2015.

36. Motoi S, Navolan DB, Malita D, Ciohat I, Nemescu D, Manciuc C, Gorun F, Vilibic-Cavlek T, Boda D, Craina M and Dobrescu A: A decreasing trend in Toxoplasma gondii seroprevalence among pregnant women in Romania-results of a large-scale study. Exp Ther Med 20: 3536-3540, 2020.
37. Olariu TR, Petrescu C, Darabus G, Lighezan R and Mazilu O: Seroprevalence of Toxoplasma gondii in Western Romania. Infect Dis (Lond) 47: 580-583, 2015.

38. Fanigliulo D, Marchi S, Montomoli E and Trombetta CM: Toxoplasma gondii in women of childbearing age and during pregnancy: Seroprevalence study in central and Southern Italy from 2013 to 2017. Parasite 27: 2, 2020.

39. Buffolano W, Gilbert RE, Holland FJ, Fratta D, Palumbo F and Ades AE: Risk factors for recent toxoplasma infection in pregnant women in Naples. Epidemiol Infect 116: 347-351, 1996.

40. Valcavi PP, Natali A, Soliani L, Montali S, Dettori G and Cheezi C: Prevalence of anti-Toxoplasma gondii antibodies in the population of the area of Parma (Italy). Eur J Epidemiol 11: 333-337, 1995.

41. Capretti MG, De Angelis M, Tridapalli E, Orlandi A, Marangoni A, Moroni A, Guerra B, Arcuri S, Marsico C and Faldella G: Toxoplasmosis in pregnancy in an area with low seroprevalence: Is prenatal screening still worthwhile? Pediatr Infect Dis J 33: 5-10, 2014.

42. Dalmartello M, Parazzini F, Pedron M, Pertile R, Collini L, La Vecchia C and Piffer S: Coverage and outcomes of antenatal tests for infections: A population based survey in the Province of Trento, Italy. J Matern Fetal Neonatal Med 32: 2049-2055, 2019.

This work is licensed under a Creative Commons Attribution-NonCommercial-NoDerivatives 4.0 International (CC BY-NC-ND 4.0) License. 